\title{
1 Cancer in adolescents and young adults living with HIV
}

2

3

$4{ }^{1}$ Institute of Social and Preventive Medicine, University of Bern, Switzerland

5

6

7

8

9

Julia Bohlius ${ }^{1}$, Caroline Foster ${ }^{2}$, Gita Naidu ${ }^{3}$, Mazvita Sengayi ${ }^{4,5}$, and Anna Turkova ${ }^{6,7 *}$

2900 and Family Clinics, Imperial College NHS Trust, London, UK Witwatersrand, Johannesburg

${ }^{4}$ National Cancer Registry, National Health Laboratory Service South Africa

${ }^{6}$ MRC CTU, University College London Institute of Clinical Trials and Methodology

${ }^{7}$ Paediatric infectious Diseases Department, Great Ormond Street Hospital, London UK

*Corresponding author:

MSc, MRC CTU, University College London Institute of Clinical Trials and Methodology, 90 High

Holborn, London, UK. Tel: +442076704658 E-mail address: a.turkova@ucl.ac.uk

\section{Acknowledgements}

\section{Financial support and sponsorship} direct role in manuscript writing or the decision to submit for publication.

${ }^{3}$ Paediatric Haematology Oncology, Chris Hani Baragwanath Academic Hospital, University of the

${ }^{5}$ School of Public Health, Faculty of Health Sciences, University of the Witwatersrand, Johannesburg,

The authors would like to thank Mary Mahy and Juliana Daher from Joint United Nations Programme on HIV/AIDS (UNAIDS) for providing number of adolescents living with HIV stratified by age group.

This work was supported by core funding to the UK Medical Research Council [MC_UU_12023/26], the National Institute of Allergy and Infectious Diseases of the National Institutes of Health [5U01Al069924-05], and the Swiss National Science Foundation [320030_169967]. The funders had no 
There are no conflicts of interest.

29

\section{Keywords}

Adolescents, cancer, HIV, prevention, young adults

\section{Key points}

- $\quad$ AYALHIV are at increased risk of AIDS and non-AIDS defining malignancies, associated with immune dysregulation and coinfection with oncogenic viruses.

- Non-Hodgkin lymphoma and Kaposi sarcoma are the commonest malignancies occurring in AYALHIV globally.

- Reducing the risk of cancer in AYALHIV requires increased access to suppressive antiretroviral therapy, HPV and HBV vaccination, screening and treatment for HBV/hepatitis C virus coinfection, and programmatic screening for cervical and anogenital cancers.

- Improvement in cancer estimates for AYALHIV requires data disaggregated by age and route of HIV transmission, which is currently lacking.

- Enabling long-term follow-up of children and adolescents living with HIV, including survivors of a dual diagnosis of HIV and malignancy as they undergo transition into adult services, requires effective linkage of pediatric and adult cohorts. 


\section{$46 \quad 3$ Tables and 1 Figure}

47 Table 1: Infection-associated cancers and related clinical HIV stage

$48 \quad$ Table 2: Search strategy for Medline (PubMed)

49 Table 3: Kaposi sarcoma incidence rate in HIV-positive adolescents and young adults who started

50 antiretroviral therapy.

51 Figure 1: Estimates for number of adolescents and young adults living with HIV in 2016 stratified by 52 age group, sex, and UNAIDS region. 
54

55

56

57

58

59

60

\section{Recent findings}

62

63

64

65

66

67

68

69

70

71

72

73 2016 and 2017.

\section{Summary}

\section{ABSTRACT}

Purpose of the review

Adults living with HIV have an increased risk of malignancy yet there is little data for adolescents and young adults. We reviewed recently published cancer epidemiology, treatment, and outcome data for adolescents and young adults living with HIV (AYALHIV) aged 10 to less than 25 years between

AYALHIV are at increased risk of developing cancer compared to their uninfected peers. Kaposi sarcoma and non-Hodgkin lymphoma occur most frequently with variation by geographical region. Increased cancer risk is associated with HIV-related immunosuppression and coinfection with oncogenic viruses. Published data, particularly on posttreatment outcomes, remain limited and analyses are hampered by lack of data disaggregation by age and route of HIV transmission.

Although data are sparse, the increased cancer risk for AYALHIV is the cause for concern and must be modified by improving global access and uptake of antiretroviral therapy, human papilloma virus (HPV) and hepatitis B virus (HBV) vaccination, screening for hepatitis B and C infection, and optimized cancer screening programs. Education aimed at reducing traditional modifiable cancer risk factors should be embedded within multidisciplinary services for AYALHIV. 


\section{INTRODUCTION}

The number of adolescents and young adults living with HIV (AYALHIV) continue to rise due to high rates of new infections and increasing life expectancy on antiretroviral therapy (ART). AYALHIV (between the ages of 10 to $<25$ years) account for $13 \%$ of those living with HIV; the majority of them are from sub-Saharan Africa (SSA). Adolescence is the only age group with a rising AIDS-related mortality [1] (Fig. 1).

\section{Adolescence, transition, and cancer risk}

Historically, malignancies in people living with HIV are categorized as AIDS-defining and non-AIDS defining (Table 1) [2,3]. AYALHIV are at increased risk of developing both AIDS and non-AIDS-defining cancers compared to HIV-negative individuals [4]. The increased cancer risk for those living with HIV is driven by interlinked immunosuppression, decreased cancer surveillance, persistent coinfection with oncogenic viruses, and HIV viremia. Immediate ART initiation, before immunosuppression occurs, significantly reduces risk of cancer $[5,6]$. However, AYALHIV have lower rates of ART uptake, increased nonadherence to ART, and higher rates of loss to follow-up compared to younger children and older adults resulting in poorly controlled HIV $[4,7 \&, 8]$.

Currently, the most frequent cancers in AYALHIV are, depending on geographic region, Kaposi sarcoma, non-Hodgkin lymphoma(NHL), and leiomyosarcoma $[4,9,10,11]$. Kaposi sarcoma is associated with human herpes virus 8 (HHV-8) infection, leiomyosarcoma, and some NHL subtypes, with Epstein-Barr virus (EBV) infection [12,13]. In American adolescents (15-19 years) living with HIV, 49\% [95\% confidence interval (CI) 39-63] of all cancers were attributable to infectious precipitants [9]. Higher rates of unprotected sex in behaviourally infected AYALHIV increase acquisition of sexually transmitted oncogenic viruses including high-risk human papilloma viruses (hrHPVs) and hepatitis B and C viruses (HBV, HCV) potentiating cervical, oropharyngeal, anogenital cancers, and hepatocellular carcinomas (HCCs), respectively [13]. Perinatally infected AYALHIV may 
face increasedriskof cancer comparedto their behaviourally infected peers due to lifelong exposure to HIV, immune dysregulation, and if coinfected perinatally with HBV and/or HCV.

Last, the period of transition of healthcare between pediatric and adult services is associated with poorer health outcomes in many chronic diseases, including HIV [14]. Global models of transition vary widely between countries, income settings, and individual diseases and an adolescent living with HIV and a previous or current cancer diagnosis may have to negotiate two independent transition processes $[15,16]$. For young people living with HIV, transition typically occurs during late teens or early 20s, an age with peak incidence in Hodgkin lymphoma diagnoses within the general population [16-18].

\section{METHODS}

We searched PubMedon November 2nd 2017 (search terms are shown in Table 2). We restricted the search to January 1st 2016 to November 1st 2017. We identified 289 references, which were reviewed by the authors. We included papers that reported cancer incidence rates, risk factors, survival, or prevention interventions in AYALHIV aged 10 to less than 25 years. Papers reporting incidence rate in adults without further age disaggregation for less than 25 year olds were not considered. We included original articles, systematic reviews, and case reports. Expert reviews were excluded. A few older important studies were used to support key statements.

\section{CANCER EPIDEMIOLOGY IN ADOLESCENTS AND YOUNG ADULTS LIVING WITH HIV}

In the recent literature, there were limited data on cancers disaggregated by age and virtually no data disaggregated by mode of HIV transmission. It was, therefore, not possible to describe differences in perinatally and horizontally infected AYALHIV. Although pediatric and adult cohorts linking is being developed $[19,20,21]$, there are only few longitudinal follow-up results for cancer risk in children and adolescents transitioning to adult care [22]. 


\section{Non-Hodgkin lymphoma}

A single-center cohort study from the United Kingdom reported on the increased risk of a new lymphoma diagnoses in young adults living with perinatally acquired HIV (PaHIV) following transition to adult care [22]. A total of 5 out of 147 (3.4\%) developed lymphoma at a median (range) age of 19 (18-23) years. Patients presented with advanced disease (Ann Arbor stage III/IV) mainly diffuse large B-cell lymphomas, a prolonged history of nonadherence, with a life time average of 14 years with detectable viraemia and a low nadir CD4 cell count [157 (90-220) cells/ml]. Small numbers precluded formal risk factor analysis; however, the NHL incidence rate significantly exceeded that of the age matched general UK population; incidence rate ratio 25.9 (95\% $\mathrm{Cl} 8.31-61.7), \mathrm{P}<0.0001$. Treatment outcomes were not reported. This study echoes a previous report from Italy, describing two cases of Burkitt lymphoma in AYALHIV who were chronically exposed to high-level HIV viremia [23]. These two case series support the concerns of longer term oncogenic risk for the current generation of perinatally infected AYALHIV who experienced prolonged viremia due to late diagnosis and have low rates of viral suppression due to previous inferior ART regimens, suboptimal dosing, nonadherence and the evolution of resistance [22]. Improved HIV diagnosis and linkage to care, adherence support and potent, and well-tolerated ART is required to achieve virological suppression. Greater awareness and prompt investigation of symptoms is needed to diagnose NHL at early stages [22].

\section{Kaposi sarcoma}

We identified one Kaposi sarcoma case series [24] and two cohort studies reporting incidence rates in AYALHIV $[25,26]$. A cohort study conducted in Uganda and Kenya reported crude Kaposi sarcoma incidence rates for AYALHIV (18-24 years) higher in ART nonusers than in ART users [13]. Incidence rates tended to be higher in young men than in women;[25] although, it is unclear whether this is explained by higher prevalence of HHV-8 [27], delayed access, and poorer adherence to ART in young men or additional factors. Another study from Malawi reported a steady increase in Kaposi 
sarcoma cases in adolescents per annum despite improved ART coverage [24]. The average annual number of Kaposi sarcoma diagnoses in children and adolescents from 2006 to $2010(n=89)$ was 17.8 cases per year, compared to 25.2 cases per year from 2011 to $2015(n=126)$ [24]. This may be explained by better Kaposi sarcoma diagnosis with the improved HIV care [24\&]. A third study reported Kaposi sarcoma incidence rates for AYALHIV (aged 16-24 years) from Europe, South Africa, North America, and Asia. In this multiregional cohort analysis adolescents in South Africa had very high Kaposi sarcoma incidence rates (303, 95\% Cl 176-523) per 100000 person years, followed by adolescents in Latin America, North America, and Europe (Table 3).

\section{Invasive cervical cancer}

Cervical cancer is the fourth leading cause of cancer incidence and mortality for women globally $[28,29]$. In AYALHIV, one cohort study reported an incidence rate for invasive cervical cancer (ICC) in young women (18-25 years) of 223 (100-496) per 100000 person years [30]. Women living with HIV have higher hrHPV prevalence [31,32] and more diverse HPV subtypes than their HIV negative counterparts [33,34,32]. HIV-infected young women have high incidence of cervical dysplasia [35];compared to HIV uninfected peers, the rate has been with reported incidence to be three times higher [36].

\section{Hepatocellular carcinoma}

HIV/HBV and HIV/HCV coinfections are associated with an increased risk of liver disease including

HCC in adults; however, there are minimal data in those coinfected either perinatally or in childhood [37]. Two cases of HCC in adolescents are described in the literature. One male, of black African origin, with PaHIV/HBV developed a rapidly progressive HCC aged 19 despite more than a decade of suppressive ART for both HBV and HIV and regular HCC screening. Despite timely surgery, he died of recurrent metastatic HCC within a year of diagnosis [37]. A second adolescent with PaHIV developed an $\mathrm{HCC}$ but with no evidence of hepatitis coinfection. He had slow disease progression despite being 
severely immunocompromised, with no evidence of recurrence more than a year from surgical resection [38].

\section{Smooth muscle tumours}

A recent study from South Africa reported a case series of EBV-associated smooth muscle tumors in AYALHIV and adults [39]. Five cases occurred in adolescents (10-15 years) with median CD4 cell count 616 (range 1-1331) cells/ml; all were female, and all but one survived [39].

\section{TREATMENT, PROGNOSIS, AND SURVIVORSHIP}

There were limited published data for cancer outcomes in AYALHIV; however, adult studies suggest disparities in access to cancer treatment and poorer outcomes in adults living with HIV compared to their uninfected peers [40]. A retrospective observational study from Malawi reported treatment outcomes for 70 children and adolescents with HIV (median age 8.6 (1.7-17.9) years) diagnosed with Kaposi sarcoma [41]. Local first-line chemotherapy included bleomycin and vincristine (BV). In 2012, doxorubicin became available in Malawi, which was added for second-line therapy. Paclitaxel was used for the third line. ART-naïve individuals started nevirapine-based ART within 2 weeks of chemotherapy. Of all patients, $28 \%$ had severe immunosuppression and nearly half were on ART at time of Kaposi sarcoma diagnosis. The combination of BV was well tolerated with ART, with minimal severe adverse events. Over half (58\%) have survived at median follow-up of 29 (15-50) months. Lymphadenopathic Kaposi sarcoma, the most common clinical presentation in children in eastern Africa, was associated with the best outcomes. Kaposi sarcoma with woody edema had a more chronic disease course, whereas visceral disease and Kaposi sarcoma with more than 20 widespread 'disseminated' skin/oral lesions were independently associated with increased mortality. Identifying risk factors associated with unfavorable outcomes may be critical to determining which patients will require alternative therapeutic strategies [41]. 
Timely ART initiation in individuals with HIV-related malignancies reduces morbidity associated with opportunistic infections and improves overall survival. However, preexisting HIV-associated organ dysfunction, coexistence of opportunistic infections, compound immunosuppression caused by HIV and chemotherapy, as well as drug interactions between ART and chemotherapy and overlapping treatment-related toxicities make management of patients with HIV and cancer complex. A recent study suggests coadministration of chemotherapy with ART based on integrase strand-transfer inhibitors or nonnucleoside reverse transcriptase inhibitors but not boosted protease inhibitors results in better safety profiles and higher suppressed viral replication [39].

Adult survivors of childhood/adolescent cancer have a lifelong increased morbidity and mortality as well as amplified risk of secondary malignancy [42]. Morbidity may be multisystem impacting on cardiorespiratory, skeletal, renal, neurocognitive, endocrine, and reproductive health compounded with significant psychosocial issues affecting mental health [43]. Annual reviews are recommended for survivors of childhood cancer for screening, prevention, and treatment of late effects; however, uptake following transition to adult care is poor [44]. AYALHIV who survived malignancy face similar issues compounded by risk of cumulative long-term sequelea of HIV. Potentially, they have an increased risk of a secondary malignancy due to their underlying immune dysregulation and require enhanced support during transition to ensure retention in care and viral suppression.

\section{PRIMARY AND SECONDARY PREVENTION}

Early HIV diagnosis and timely ART may substantially reduce the risk of AIDS-defining cancers $[5,6,10,11,45]$. Unlike for HPV, HBV, and HCV, there are no vaccines or specific treatment for EBV and HHV-8, and early access to suppressive ART remains the most important preventive measure for cancers related to these infections. 


\section{High-risk variants of human papilloma virus}

The high global prevalence of persistent hrHPV infection in both female and male AYALHIV $[33,34,46,47,35]$ and high proportion of high-grade precancerous lesions [35] highlight the importance of sex-neutral HPV vaccination. HPV vaccination induces good HPV-specific cellmediated immune responses in AYALHIV, compared to HIV-uninfected age-matched controls; although, three rather than two doses are still recommended for AYALHIV due to a data gap [48]. Currently, only $11(6 \%)$ countries vaccinate males in their national immunization programs [49]. WHO and American Society of Clinical Oncology (ASCO) guidance prioritize vaccination of girls based on cost-effectiveness analyses for prevention of cervical cancer; boys can be included if the vaccine uptake among priority female population is less than $50 \%$ and resources are available $[49,50]$. A relatively high proportion of hrHPV types in young women in SSA are not covered by currently available HPV vaccines, [31,51] which supports early initiation of cervical screening for all sexually active women living with HIV irrespective of age as recommended by WHO and ASCO [49,52]. A study from Saudi Arabia showed that male circumcision may play role in reduction of HPV infection, penile cancers, and cervical cancer among women with circumcised partners [53]. Screening for anal cancers is not routinely recommended; although, some experts suggest that this might be effective $[54,55]$. There is an urgent need of prospective studies validating different approaches for prevention and screening of cervical and anogenital cancers.

\section{Hepatitis B and C}

Occurrence of HCC early in adulthood underlines the importance of primary prevention with HBV vaccination and screening for chronic HBV and HCV coinfection. Systematic screening for HBV and HCV infection is limited in most sub-Saharan African countries [56]. Hepatitis B vaccination from birth with serological monitoring and boosting when appropriate, and education around prevention of HCV acquisition should be embedded within the life span care of those living with HIV. There is no consensus on HCC screening; although, 6 monthly liver ultrasounds and alpha-fetoprotein are 
supported by WHO guidance [38,57]. Reducing risk of HCC includes HBV viral suppression with tenofovir-based regimens, avoidance of excessive alcohol and weight optimization. Increased advocacy for rapid access to curative direct-acting antivirals for HCV for coinfected adolescents is urgently required.

Knowledge, awareness, and uptake of sexual and reproductive health services (SRS) is insufficient among young people $[58,59]$. Enhanced counselling, integration, or linkage to SRS can improve the uptake of voluntary male circumcision and cervical cancer screening [60]. AYALHIV require access to 'youth friendly' SRS integrated within multidisciplinary HIV care that includes primary prevention packages addressing vaccination, ART adherence, smoking [33], alcohol and substance use [61], weight management, and where appropriate screening for HPV, HBV, and HCV-related malignancies.

\section{CONCLUSION}

People living with HIV, including adolescents and young adults, are at increased risk of malignancy, due to immune dysregulation and the persistence of oncogenic viruses. While the excess cancer risk is reduced with suppressive ART, ART coverage is still suboptimal in many settings, and AYALHIV have the lowest rates of engagement with each aspect of the HIV care cascade. Improving HIV diagnosis, linkage, and retention in care on sustained suppressive ART for AYALHIV remains the most important cancer preventive measure. However, this must go hand in hand with integrated cancer screening and education programs including prevention of traditional cancer modifiable risk factors for a vulnerable population who currently face an increased life time risk of malignancy, while they negotiate their transition to adulthood living with HIV. Increased awareness among healthcare workers and prompt investigation of suggestive symptoms is needed to diagnose cancers at early stages. In the era of effective ART, AYALHIV should have access to cancer treatment and supportive care compared to their uninfected peers. 


\section{REFERENCES}

1. UNAIDS: UNAIDS 2017 estimates [Internet]. [Accessed 15 December 2017].

2. Centers for Disease Control and Prevention (CDC). Revised surveillance case definition for HIV infection-United States, 2014. MMWR Recomm Rep 2014; 63:1-10.

3. World Health Organization: WHO case definitions of HIV for surveillance and revised clinical staging and immunological classification of HIV-related disease in adults and children August 2006. [Accessed 15 December 2017]

4. Simard EP, Shiels MS, Bhatia K, Engels EA. Long-term cancer risk among people diagnosed with AIDS during childhood. Cancer Epidemiol Biomarkers Prev 2012; 21:148-154.

5. Borges $A^{\prime} H$. Neuhaus J, Babiker AG, et al. Immediate antiretroviral therapy reduces risk of infection-related cancer during early HIV infection. Clin Infect Dis 2016; 63:1668-1676.

6. Borges $\mathrm{AH}$. Combination antiretroviral therapy and cancer risk. Curr Opin HIVAIDS 2017; 12:12-19.

This is a comprehensive review of the recent cohort and randomized data on effects of ART on cancer risk in adults.

7. Enane LA, Vreeman RC, Foster C. Retention and adherence: global challenges for the longterm care of adolescents and young adults living with HIV. Curr Opin HIV AIDS 2018; 13:212-219.

This is a comprehensive review of the last 18 months data on retention in care and adherence to ART in AYALHIV.

8. Zanoni BC, Archary M, Buchan S, et al. Systematic review and meta-analysis of the adolescent HIV continuum of care in South Africa: the cresting wave. BMJ Glob Heal 2016; 1:e000004.

9. de Martel C, Shiels MS, Franceschi S, et al. Cancers attributable to infections among adults with HIV in the United States. AIDS 2015; 29:2173-2181.

10. Bohlius J, Maxwell N, Spoerri A, et al. Incidence of AIDS-defining and other cancers in HIVpositive children in South Africa: record linkage study. Pediatr Infect Dis J 2016; 35:e164e170.

11. Bohlius J; Pediatric AIDS-Defining Cancer Project Working Group for leDEA Southern Africa, TApHOD, and COHERE in EuroCoord. Kaposi sarcoma risk in HIV-infected children and adolescents on combination antiretroviral therapy from sub-Saharan Africa, Europe, and Asia. Clin Infect Dis 2016; 63:1245-1253.

12. La Ferla L, Pinzone MR, Nunnari G, et al. Kaposi' s sarcoma in HIV-positive patients: the state of art in the HAART-era. Eur Rev Med Pharmacol Sci 2013; 17:2354-2365.

13. IARC Working Group on the Evaluation of Carcinogenic Risks to Humans. Biological agents. Volume 100 B. A review of human carcinogens. IARC Monogr Eval Carcinog Risks Hum 2012; 100:1-441. 
14. Sohn AH, Vreeman RC, Judd A. Tracking the transition of adolescents into adult HIV care: a global assessment. J Int AIDS Soc 2017; 20:1-3.

This is a special edition of the Journal of the International AIDS Society with 12 articles dedicated to describing the global issues faced during transition of HIV care by income setting and by route of HIV acquisition, highlighting data gaps and future research and services needs.

15. Foster C, Fidler S. Optimising HIV transition services for young adults. Curr Opin Infect Dis 2018; 31:33-38.

This is a comprehensive review of the last 18 months data on transition from pediatric to adult care and the complex issues faced for AYALHIV both perinatally and behaviorally infected.

16. Ferrari A, Barr RD. International evolution in AYA oncology: current status and future expectations. Pediatr Blood Cancer 2017; 64:e26528.

17. Grubb WR, Neboori HJ, Diaz AD, et al. Racial and ethnic disparities in the pediatric Hodgkin lymphoma population. Pediatr Blood Cancer 2016;63:428-435.

18. Smith A, Crouch S, Lax S, et al. Lymphoma incidence, survival and prevalence 2004-2014: sub-type analyses from the UK's Haematological Malignancy Research Network. Br J Cancer 2015; 112:1575-1584.

19. Writing group for the Kids to Adults Working Group and Data Management and Harmonisation Group in EuroCoord. Children and young people with perinatal HIV in Europe: epidemiological situation in 2014 and implications for the future. Euro Surveill 2016; 21:30162.

20. Davies MA, Tsondai P, Tiffin N, et al. Where do HIV-infected adolescents go after transfer? Tracking transition/transfer of HIV-infected adolescents using linkage of cohort data to a health information system platform. J Int AIDS Soc 2017; 20:21668.

The paper highlights the complexities in linking pediatric and adult HIV surveillance systems to enable accurate data collection on long-term outcomes for those who have acquired HIV in childhood and adolescence.

21. Judd A, Collins IJ, Parrott F, et al. Growing up with perinatal HIV: changes in clinical outcomes before and after transfer to adult care in the UK. J Int AIDS Soc 2017; 20:21577.

One of the very few studies of national outcome data for perinatally infected youth following transition to adult care and highlights that for some adolescents the issues with adherence to ART occur prior to transition.

22. Eades $C P$, Herbert SA, Edwards SG, et al. High rate of lymphoma among a UK cohort of adolescents with vertically acquired HIV-1 infection transitioning to adult care in the era of antiretroviral therapy. AIDS 2015;30:153-156.

23. Zangari $P$, Santilli $V$, Cotugno N, et al. Raising awareness of non-Hodgkin lymphoma in HIVinfected adolescents: report of 2 cases in the HAART era. J Pediatr Hematol Oncol 2013; 35:e134-e137. 
24. El-Mallawany NK, Villiera J, Kamiyango W, et al. Increasing numbers of new Kaposi sarcoma diagnoses in HIV-infected children and adolescents despite the wide availability of antiretroviral therapy in Malawi. Clin Infect Dis 2017;64:818-819.

This case series of Kaposi sarcoma cases at pediatric HIV-related malignancy program in Lilongwe, Malawi, showed an increase in annual number of new cases in the time period 2006-2015.

25. Semeere $A$, Wenger $M, B$ Busakhala $N$, et al. A prospective ascertainment of cancer incidence in sub-Saharan Africa: the case of Kaposi sarcoma. Cancer Med 2016; 5:914-928.

26. Bohlius J; AIDS-defining Cancer Project Working Group for leDEA and COHERE in EuroCoord. Comparison of Kaposi sarcoma risk in HIV-positive adults across five continents: a multiregional multicohort study. Clin Infect Dis 2017; 65:1316-1326.

This is an international multiregional cohort study directly comparing Kaposi sarcoma risk in HIV-positive adults in Southern Africa, Europe, North, and Latin America. Data for young adults aged 18-25 years are reported separately.

27. Begre' L, Rohner E, Mbulaiteye SM, et al. Is human herpesvirus 8 infection more common in men than in women? Systematic review and meta-analysis. Int J cancer 2016; 139:776-783.

28. Torre LA, Bray F, Siegel RL, et al. Global cancer statistics, 2012. CA Cancer J Clin 2015; 65:87108.

29. Torre LA, Siegel RL, Ward EM, Jemal A. Global cancer incidence and mortality rates andtrends:an update.Cancer EpidemiolBiomarkersPrev2016; 25:16-27.

30. Rohner E, Sengayi M, Goeieman B, et al. Cervical cancer risk and impact of Pap-based screening in HIV-positive women on antiretroviral therapy in Johannesburg, South Africa. Int J Cancer 2017; 141:488-496.

This is a single cohort study with cervical cancer cases identified through probabilistic record linkage with theNationalCancerRegistry in South Africa.Cervical cancer incidence rate for young women aged 18-25 years is reported separately.

31. Mbatha JN, Taylor M, Kleppa E, et al. High-risk human papillomavirus types in HIV-infected and HIV-uninfected young women in KwaZulu-Natal, South Africa: implications for vaccination. Infect Dis (Auckl) 2017; 49:601-608.

32. Mbulawa ZZ, Coetzee D, Williamson AL. Human papillomavirus prevalence in South African women and men according to age and human immunodeficiency virus status. BMC Infect Dis 2015; 15:459.

33. Ursu RG, Onofriescu M, Luca A, et al. The need for cervical cancer control in HIV-positive and HIV-negative women from Romania by primary prevention and by early detection using clinically validated HPV/DNA tests. PLoS One 2015; 10:e0132271.

34. Mbatha JN, Taylor M, Kleppa E, et al. High-risk human papillomavirus types in HIV-infected and HIV-uninfected young women in KwaZulu-Natal, South Africa: implications for vaccination. Infect Dis (Auckl) 2017; 49:601-608. 
This cross-sectional study of HPV infection in sexually active young women recruited from high schools showed that the HPV prevalence was significantly higher in women with HIV. Four of the most predominant high-risk HPV types were not covered by any of the existent HPV vaccines.

35. Silva L, Miranda A, Batalha R, et al. High-risk human papillomavirus and cervical lesions among women living with HIV/AIDS in Brazilian Amazon, Brazil. Braz J Infect Dis 2015; 19:557-562.

36. Gaym A, Mashego M, Kharsany ABM, et al. High prevalence of abnormal Pap smears among young women co-infected with HIV in rural South Africa -implications for cervical cancer screening policies in high HIV prevalence populations. S Afr Med J 2007; 97:120-123.

37. Seers $T$, Sarker $D$, Ross $P$, et al. Hepatocellular carcinoma in perinatally acquired HIV and HBV Coinfection: a case report. Pediatr Infect Dis J 2017;36:1156-1158.

The only case report of HCC in perinatal HBV/HIV coinfection with a review of the literature on HCC screening in coinfected populations.

38. Venkataramani $\mathrm{M}$, Hutton $\mathrm{N}$, Colombani $\mathrm{P}$, et al. Hepatocellular carcinoma in a teenager with perinatally acquired HIV Infection without hepatitis B or C coinfection: a case report. AIDS Patient Care STDS 2010; 24:693-696.

39. Pather S, Wainwright RD, Sahid F, et al. Human immunodeficiency virusrelated Epstein-Barr virus-associated smooth muscle tumours: South African experience from Chris Hani Baragwanath Academic Hospital. South African J Infect Dis 2017; 0053:1-4.

40. Cingolani A, Lepri AC, Teofili L, et al. Survival and predictors of death in people with HIVassociated lymphoma compared to those with a diagnosis of lymphoma in general population. PLoS One 2017; 12:1-15.

The study showed a reduced survival in HIV-infected patients with Hodgkin lymphoma, a non-AIDS defining cancer, compared to HIV-uninfected patients after adjusting for key potential confounders.

41. El-Mallawany NK, Kamiyango W, Slone JS, et al. Clinical factors associated with long-term complete remission versus poor response to chemotherapy in HIV-infected children and adolescents with Kaposi sarcoma receiving bleomycin and vincristine: a retrospective observational study. PLoS One 2016;11:e0153335.

42. Lee JS, DuBois SG, Coccia PF, et al. Increased risk of second malignant neoplasms in adolescents and young adults with cancer. Cancer 2016;122:116-123.

43. Overholser L, Kilbourn K, Liu A. Survivorship issues in adolescent and young adult oncology. Med Clin North Am 2017; 101:1075-1084.

The review highlights the unique survivorship issues that adolescents and young adult cancer survivors face. Fertility, reproductive health, and psychosocial issues are especially important.

44. Wong AWK, Chang TT, Christopher K, et al. Patterns of unmet needs in adolescent and young adult (AYA) cancer survivors: in their own words. J Cancer Surviv 2017; 11:751-764. 
45. Kelly $\mathrm{H}$, Weiss HA, Benavente $\mathrm{Y}$, et al. Association of antiretroviral therapy with high-risk human papillomavirus, cervical intraepithelial neoplasia, and invasive cervical cancer in women living with HIV: a systematic review and meta-analysis. Lancet HIV 2018; 5:e45-e58. This was the first meta-analysis assessing the effect of ART on high-risk HPV infection, cervical lesions, and cervical cancer in adults. The results showed that ART reduces progression of precancerous cervical lesions and incidence of invasive cervical cancer.

46. Ngabo F, Franceschi S, Baussano I, et al. Human papillomavirus infection in Rwanda at the moment of implementation of a national HPV vaccination programme. BMC Infect Dis 2016; $16: 225$

47. Nowak RG, Gravitt PE, He X, et al. Prevalence of anal high-risk human papillomavirus infections among HIV-positive and HIV-negative men who have sex with men in Nigeria. Sex Transm Dis 2016; 43:243-248.

48. Rainone $V$, Giacomet $V$, Penagini $F$, et al. Human papilloma virus vaccination induces strong human papilloma virus specific cell-mediated immune responses in HIV-infected adolescents and young adults. AIDS 2015;29:739-743.

49. World Health Organization. Human papillomavirus vaccines: WHO position paper, May 2017-recommendations. Vaccine 2017; 35:5753-5755.

This is an updated WHO position paper on HPV vaccination for national programs. The paper summarizes background information on HPV infection and currently available HPV vaccines and outlines evidence-based recommendations endorsed by the WHO Strategic Advisory Group of Experts on immunization.

50. Arrossi S, Temin S, Garland S, et al. Primary prevention of cervical cancer: American Society of Clinical Oncology Resource-Stratified Guideline. J Glob Oncol 2017; 3:611-634.

The paper presents resource-stratified, evidence-based recommendations on the primary prevention of cervical cancer produced by a multidisciplinary, multinational panel of experts which was convened by the ASCO.

51. Edna Omar V, Orvalho A, Na' lia I, et al. Human papillomavirus prevalence and genotype distribution among young women and men in Maputo city, Mozambique. BMJ Open 2017; 7:e015653.

52. Jeronimo J, Castle PE, Temin S, et al. Secondary prevention of cervical cancer: ASCO Resource-Stratified Clinical Practice Guideline. J Glob Oncol 2017; 3:635-657.

The paper presents resource-stratified, evidence-based recommendations on the secondary prevention of cervical cancer produced by a multidisciplinary, multinational panel of experts which was convened by the ASCO.

53. Alkhenizan A, Elabd K. Nontherapeutic infant male circumcision. Evidence, ethics, and international law perspectives. Saudi Med J 2016;37:941-947.

54. Gosens KC, Richel O, Prins JM. Human papillomavirus as a cause of anal cancer and the role of screening. Curr Opin Infect Dis 2017; 30:87-92. 
This paper reviewed screening approaches and treatment of anal cancer in HIV positive men.

55. Esser S, Kreuter A, Oette M, et al. German-Austrian guidelines on anal dysplasia and anal cancer in HIV-positive individuals: prevention, diagnosis, and treatment. J Dtsch Dermatol Ges 2015; 13:1302-1319.

56. Coffie PA, Egger M, Vinikoor MJ, et al. Trends in hepatitis B virus testing practices and management in HIV clinics across sub-Saharan Africa. BMC Infect Dis 2017; 17:706.

This is a medical chart review in 12 large HIV clinics in nine sub-Saharan countries. The results showed limited systematic HBV screening in all clinics except in a clinic in South Africa and extremely limited screening for HCV infection. Majority of HIV/HBV-coinfected patients (90\%) were initiated on tenofovir-containing ART.

57. World Health Organization: Guidelines for the Prevention, Care and Treatment of Persons with Chronic Hepatitis B Infection. March 2015 [Accessed 15 December 2017].

58. Lin YJ, Fan LW, Tu YC. Perceived risk of human papillomavirus infection and cervical cancer among adolescent women in Taiwan. Asian Nurs Res (Korean Soc Nurs Sci) 2016; 10:45-50.

59. Gerend MA, Madkins K, Phillips G 2nd, Mustanski B. Predictors of human papillomavirus vaccination among young men who have sex with men. Sex Transm Dis 2016; 43:185-191.

60. Hewett PC, Nalubamba M, Bozzani F, et al. Randomized evaluation and costeffectiveness of HIV and sexual and reproductive health service referral and linkage models in Zambia. BMC Public Health 2016; 16:785.

61. Kahn JA, Rudy BJ, Xu J, et al. Prevalence and risk factors for oral DNA tumor viruses in HIVinfected youth. J Med Virol 2016; 88:1944-1952. 
Table 1

Infection-associated cancers and related clinical HIV stage does not classify leiomyosarcoma [3]. cancer have been linked to HIV infection and included in the group of non-AIDS-defining illnesses.

501 virus; HHV-8, human herpes virus-8.

\begin{tabular}{|c|c|c|}
\hline Malignancy & $\begin{array}{l}\text { Association with } \\
\text { on cogenic viruses }\end{array}$ & $\begin{array}{c}\text { HIV } \\
\text { staging }^{a}\end{array}$ \\
\hline \multicolumn{3}{|l|}{ AIDS-defining malignancies } \\
\hline \multicolumn{3}{|l|}{ Non-Hodgkin lymphoma } \\
\hline $\begin{array}{l}\text { Burkitt's lymphoma } \\
\text { Large cell (immunoblastic) } \\
\text { lymphoma } \\
\text { Primary central nervous } \\
\text { system lymphoma }\end{array}$ & $\begin{array}{l}\text { EBV } \\
\text { EBV }\end{array}$ & $\begin{array}{l}\text { CDC C } \\
\text { WHO } 4\end{array}$ \\
\hline Kaposi sarcoma & HHV-8 & $\mathrm{CDC} C, \mathrm{WHO} 4$ \\
\hline Invasive cervical carcinoma & HPV & $\mathrm{CDC} C, \mathrm{WHO} 4$ \\
\hline \multicolumn{3}{|l|}{ Non-AIDS-defining malignancies ${ }^{b}$} \\
\hline \multicolumn{3}{|l|}{ Smooth muscle tumours } \\
\hline $\begin{array}{l}\text { Leiomyoma (benign) } \\
\text { Leiomyosarcoma (malignant) }\end{array}$ & $\begin{array}{l}\text { EBV } \\
\text { EBV }\end{array}$ & $\mathrm{CDCB}$ \\
\hline Hodgkin lymphoma & EBV & - \\
\hline Hepatocellular carcinoma & $\mathrm{HBV}, \mathrm{HCV}$ & - \\
\hline Anal cancer & HPV & - \\
\hline
\end{tabular}


Search strategy for Medline (PubMed)

506 (('Neoplasms'[Mesh]) OR (neoplasm*[Title/Abstract] OR cancer*[Title/Abstract] OR carcinoma*[Title/Abstract] OR tumor*[Title/Abstract] OR Tumor*[Title/Abstract] OR malignanc*[Title/Abstract] OR leukemic*[Title/Abstract] OR leukemic*[Title/Abstract] OR hematopoietic stem cell transplantation*[Title/Abstract] OR hematopoietic stem cell transplantation*[Title/Abstract] OR hematopoietic cell transplantation*[Title/Abstract] OR

511 hematopoietic cell transplantation[Title/Abstract])) AND

512 (('Adolescent'[Mesh]) OR ('Young Adult'[Mesh]) OR (adolescen*[Title/Abstract] OR

513 juvenile*[Title/Abstract] OR youth*[Title/Abstract] OR teen*[Title/Abstract] OR

514 underage*[Title/Abstract] OR underage[Title/Abstract] OR pubescen*[Title/Abstract] OR young

515 adult*[Title/Abstract])) AND (Search HIV Infections[MeSH] OR HIV[MeSH] OR hiv[tw] OR HIV-1*[tw]

516 OR HIV-2*[tw] OR HIV1[tw] OR HIV2[tw] OR HIV infect*[tw] OR human immunodeficiency virus[tw]

517 OR human immunedeficiency virus[tw] OR human immuno-deficiency virus[tw] OR human immune-

518 deficiency virus[tw] OR ((human immune*) AND (deficiency virus[tw])) OR acquired

519 immunodeficiency syndrome[tw] OR acquired immunedeficiency syndrome[tw] OR acquired

520 immuno-deficiency syndrome[tw] OR acquired immune-deficiency syndrome[tw] OR ((acquired

521 immune*) AND (deficiency syndrome[tw])) OR 'sexually transmitted diseases, viral' $[\mathrm{MH}]$ ) 


\section{Table 3}

524 Table 3: Kaposi sarcoma incidence rate in HIV-positive adolescents and young adults who started

525 antiretroviral therapy.

\begin{tabular}{llcc}
\hline Study & Country/region & Age group lyears] & Rate $(95 \%$ CI) per 100,000 person-years \\
\hline Semeere et al. [25] & Uganda, Kenya & $18-19$ & $245(79-760)$ \\
& Uganda, Kenya & $20-24$ & $323(245-426)$ \\
Rohner et al. [30"-] & South Africa & $16-25$ & $303(176-523)$ \\
& Latin America & $16-25$ & $248(141-438)$ \\
& North America & $16-25$ & $95(36-253)$ \\
& Europe & $16-25$ & $115(93-143)$ \\
\hline
\end{tabular}

527 
FIGURE

\section{$529 \quad$ Figure 1}

530 Estimates for number of adolescents and young adults living with HIV in 2016 stratified by age

531 group, sex, and UNAIDS region. Estimates for (i) western, central Europe and North America and (ii)

532 eastern Europe and central Asia for adolescents aged 10 to $<15$ years are not available.

533 F, female; $M$, male. Axis Y: Number of adolescents and young adults. Axis X: UNAIDS regions. Source:

534 UNAIDS 2017 estimates [1].

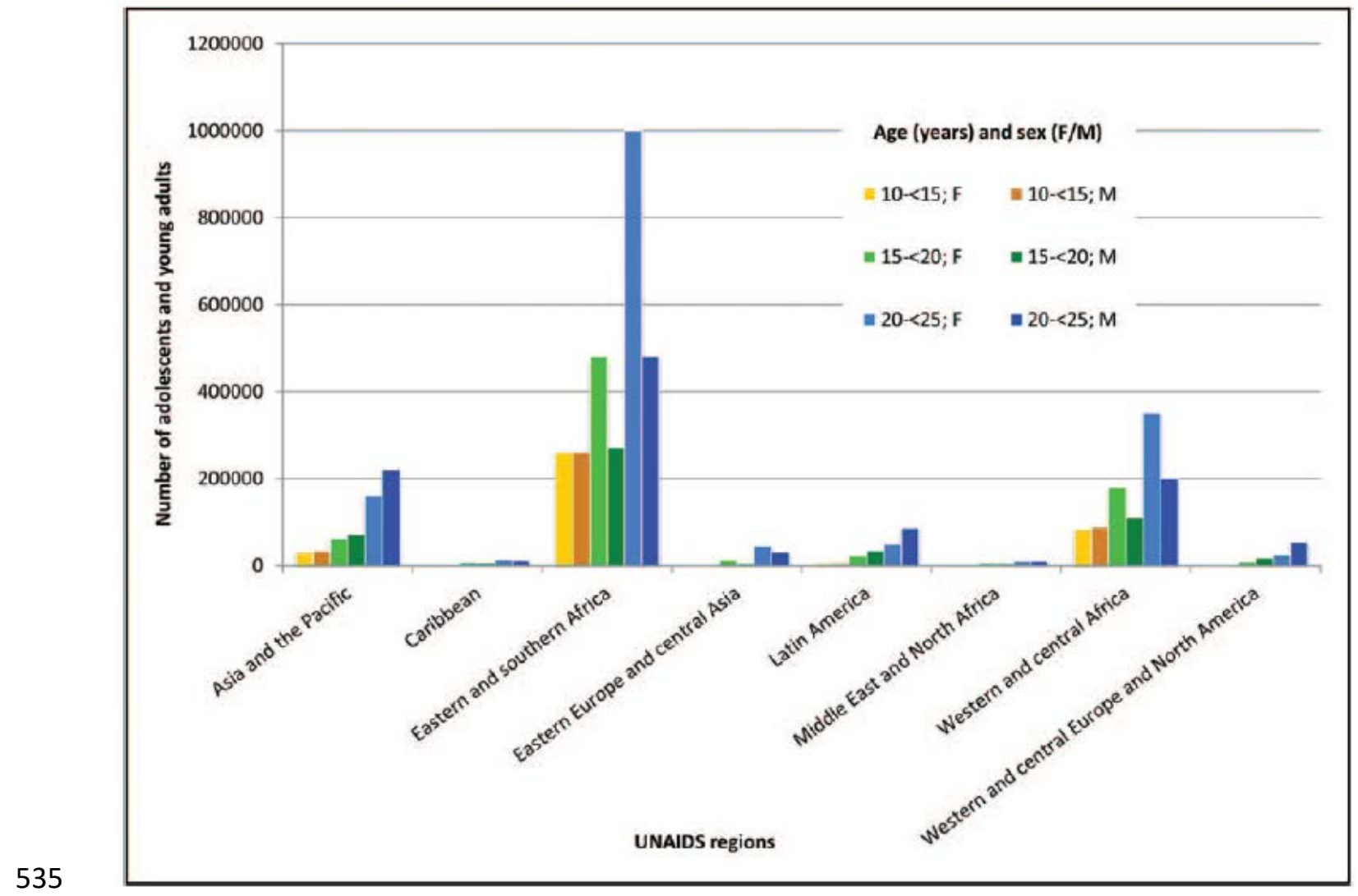

